\title{
Erratum to: Effects of preoperative and intraoperative glucose administration on glucose use and fat catabolism during laparotomy under sevoflurane anesthesia in fasted rats
}

\author{
Yoshiteru Mori ${ }^{1}$ - Takayuki Kitamura ${ }^{2} \cdot$ Gaku Kawamura $^{1} \cdot$ Kanako Sato $^{2}$ • \\ Rui Sato $^{1} \cdot$ Yuko Araki $^{1} \cdot$ Yoshitsugu Yamada $^{1}$
}

Published online: 8 October 2015

(C) The Physiological Society of Japan and Springer Japan 2015

\section{Erratum to: J Physiol Sci}

DOI 10.1007/s12576-015-0390-7

In the original publication of the article, Fig. $2 \mathrm{c}$ was published incorrectly. This error was caused during the production process. The correct version of Fig. 2 is published with this erratum.

The online version of the original article can be found under doi:10.1007/s12576-015-0390-7.

Gaku Kawamura

gaku-kawa@umin.ac.jp

1 Department of Anesthesiology, Faculty of Medicine, The University of Tokyo, 7-3-1 Hongo, Bunkyo-ku,

Tokyo 113-8655, Japan

2 Department of Anesthesiology, Toho University Sakura Medical Center, Sakura, Chiba, Japan
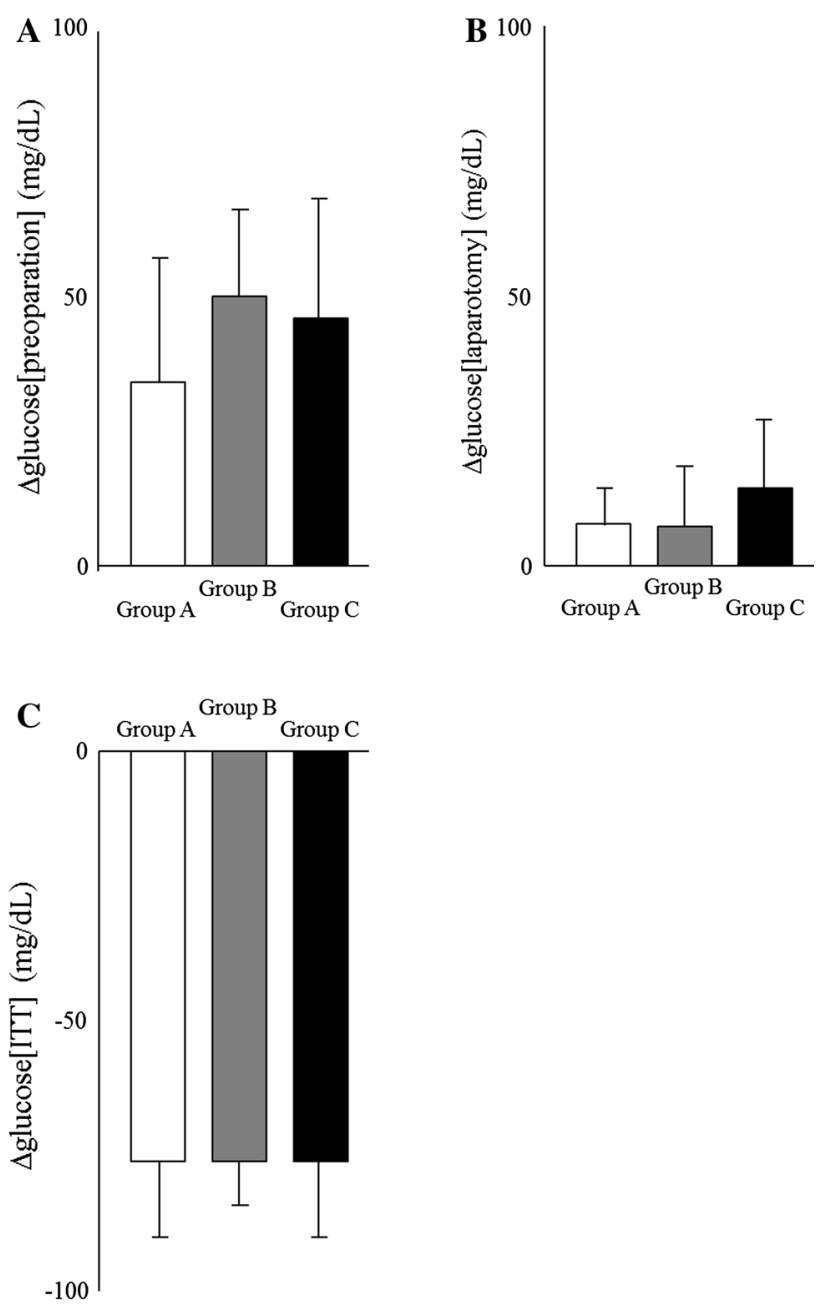

Fig. 2 Changes in blood glucose levels during the experiments. a Increases in blood glucose levels during preparations $[\Delta$ glucose (preparations)]; there was no significant difference among the three groups $(P>0.05,1$-way ANOVA). b Increases in blood glucose levels during laparotomy $[\Delta$ glucose (laparotomy)]; there was no significant difference among the three groups $(P>0.05,1$-way ANOVA). c Decreases in blood glucose levels during the insulin tolerance test $[\Delta$ glucose (ITT)]; there was no significant difference among the three groups $(P>0.05,1$-way ANOVA) 